\title{
CORRIGENDUM
}

\section{Effective mixing and counting in trees - CORRIGENDUM}

\author{
SANGHOON KWON \\ doi:10.1017/etds.2016.28, Published by Cambridge University Press, \\ 4 July 2016
}

Given a locally finite simplicial tree $\mathcal{T}$, the term weighted spectral gap (WSG) is defined in the paper [3], for a pair $(\Gamma, F)$ of a full discrete subgroup $\Gamma$ of $\operatorname{Aut}(\mathcal{T})$ and a $\Gamma$-invariant potential $F$. If $(\Gamma, F)$ has a WSG, then the exponential mixing property of the geodesic translation map $\phi: \Gamma \backslash S \mathcal{T} \rightarrow \Gamma \backslash S \mathcal{T}$ with respect to the measure $m_{\Gamma, F}^{\nu^{-}, v^{+}}$holds whenever the length spectrum of $\Gamma$ is not arithmetic.

It was claimed in Example 4.1 in [3] that geometrically finite groups have a WSG for any constant potential. However, that statement is misleading because a geometrically finite discrete group in $\operatorname{Aut}(\mathcal{T})$ can never be full unless it is convex cocompact. Nevertheless, it is proved in [2] that the exponential mixing property of the discrete time geodesic flow still holds. Their argument contains a new method of coding the discrete time geodesic flow without assuming the group $\Gamma$ is full and a suitable application of the Young's tower method, introduced in [4].

Acknowledgements. The author deeply regrets this error and thanks Frédéric Paulin for pointing it out to him. He would like to take this opportunity to mention that when $\Gamma$ is a lattice of linear groups, Theorem 1.1 can also be obtained from [1].

\section{REFERENCES}

[1] J. Athreya, A. Ghosh and Prasad. Ultrametric logarithm laws, II. Monatsh. Math. 167 (2012), 333-356.

[2] A. Broise-Alamichel, J. Parkkonen and F. Paulin. Equidistribution and counting under equilibrium states in negatively curved spaces and graphs of groups. Applications to non-Archimedean Diophantine approximation. Preprint, 2016, arXiv:1612.06717.

[3] S. Kwon. Effective mixing and counting in Bruhat-Tits trees. Ergod. Th. \& Dynam. Sys. doi:10.1017/etds.2016.28. Published online 4 July 2016.

[4] L.-S. Young. Recurrent times and rates of mixing. Israel J. Math. 110 (1999), 153-188. 